\title{
Application of Creep and Fatigue Tests on Hot Asphalt Mixes
}

Badr amousa, Mohamed E. Abdel-Motaleb

\begin{abstract}
An asphalt concrete pavement should be designed to provide a durable, skid resistance surface under in service conditions. Also, it is essential to minimize cracking and rutting in the asphalt concrete layers. To fully utilize each material in an economical design, a pavement should generally have a reasonable blanaced design between the rutting and fatigue modes of distress. The purpose of this paper is to examine both fatigue and deformation characteristics of four types of asphalt concrete mixes produced by different mixing procedure. Fatigue properties are measured using a rectangular slab specimens resting on a rubber (elastic) subgrade using the wheel tracking machine under dynaminc loading condition. Deformation characteristics of the mixes are determined using the shell creep test for 1 hour at ambient temperature. The contact stress is taken in tlie range of 6.25 psi to 100 psi which simulate a wide range of tire pressure. The results of this study indicate that, under high stress level the mixes of higher Marshal stiffness show higher resistance to creep deformation and fatigue cracking. While under low stress level the mixes of higher Marshal stability show higher resistance to creep deformation. For all investigated mixes, the stress - strain relationships are almost linear under stress-level below 25 psi for all test loading times. While under high stress levels, between 25 psi and 50 psi the rate of increase in creep strain is significantly greater than that from 50 psi to 100 psi
\end{abstract}

